Quasi-Equilibria in Markets with Non-Convex Preferences

Author(s): Ross M. Starr

Source: Econometrica, Vol. 37, No. 1 (Jan., 1969), pp. 25-38

Published by: The Econometric Society

Stable URL: http://www.jstor.org/stable/1909201

Accessed: 19/07/2011 14:39

Your use of the JSTOR archive indicates your acceptance of JSTOR's Terms and Conditions of Use, available at http://www.jstor.org/page/info/about/policies/terms.jsp. JSTOR's Terms and Conditions of Use provides, in part, that unless you have obtained prior permission, you may not download an entire issue of a journal or multiple copies of articles, and you may use content in the JSTOR archive only for your personal, non-commercial use.

Please contact the publisher regarding any further use of this work. Publisher contact information may be obtained at http://www.jstor.org/action/showPublisher?publisherCode=econosoc.

Each copy of any part of a JSTOR transmission must contain the same copyright notice that appears on the screen or printed page of such transmission.

JSTOR is a not-for-profit service that helps scholars, researchers, and students discover, use, and build upon a wide range of content in a trusted digital archive. We use information technology and tools to increase productivity and facilitate new forms of scholarship. For more information about JSTOR, please contact support@jstor.org. 


\title{
QUASI-EQUILIBRIA IN MARKETS WITH NON-CONVEX PREFERENCES ${ }^{1}$
}

\author{
BY ROSS M. STARR ${ }^{2}$
}

\begin{abstract}
A pure exchange economy is considered without the assumption of convex preferences. It is shown that the divergence from equilibrium due to non-convexity is bounded in a fashion independent of the number of traders. For a sufficiently large number of traders there are configurations arbitrarily close to equilibrium.
\end{abstract}

\section{INTRODUCTION}

THE NOTION of perfect competition as generally stated in economic literature involves a market with a "very large" number of traders, each of whom has at most a "negligible" effect on the eventual outcome of the trading process. The mathematical literature on general equilibrium has taken less than full advantage of this concept. The classic mathematical approaches to the proof of existence of general equilibrium (e.g., [9]) have assumed that every consumer maximizes utility under budget constraint, regardless of the number of consumers in the market. Thus, they have not taken advantage of the friction that has been built into the system. If individual traders are really negligible we should be just as interested in a situation where all but a few traders maximize subject to constraint as we are in the case where they all maximize. The actions of a small number of negligible individuals or the small actions of a large number of negligible individuals should be negligible.

One result of this mathematician's strictness is the insistence on the convexity of individual preferences. For in order to apply the various fixed-point theorems, which are used to prove the existence of equilibrium, it is required that excess demand functions be continuous (for the Brouwer theorem) or that excess demand correspondences be convex (for the Kakutani theorem). Strictly speaking, these will generally be the case only when individual preference sets are convex. It will be shown, however, that if the number of traders is sufficiently large there is a configuration under which the divergence from equilibrium can be made small relative to the size of the market.

The motivation for the elimination of the convexity assumption, aside from its being less than absolutely essential, is that it postulates away all forms of indivisi-

${ }^{1}$ The major portion of the work presented here was done at Stanford University. This version embodies improvements made both there and at The RAND Corporation since originally issued under ONR contract Nonr-225(50)(NR-047-004). Any views expressed in this paper are those of the author. They should not be interpreted as reflecting the views of The RAND Corporation or the official opinion or policy of any of its governmental or private research sponsors.

${ }^{2}$ I am greatly indebted to Professor K. J. Arrow for his perceptive suggestions, and to Professor R. J. Aumann and Dr. L. S. Shapley for suggestions and criticisms which have helped to make rigorous the central arguments of this paper. 
bilities and a class of relations one might call anticomplementarities. ${ }^{3}$ After all, one may be indifferent between an automobile and a boat, but in most cases one can neither drive nor sail the combination of half boat, half car. ${ }^{4}$

\section{THE MODEL}

The model used here is patterned after that of McKenzie [9]. A pure exchange economy is treated, though I think it is clear that whenever an equilibrium exists in the convex case with production, the introduction of production to the non-convex case leaves the situation virtually unaltered.

There are $n$ commodities. A commodity bundle is a vector in the nonnegative orthant $\Omega$ of $E^{n}$. Every trader $t$ has a transitive, reflexive, complete preference relation $\succsim_{t}$ defined on all the commodity bundles. Assume

Assumption A-CONTINUITY:For any $y$ in $\Omega$, the sets $\left\{x: x \succsim_{t} y\right\}$ and $\left\{x: y \succsim_{t} x\right\}$ are closed.

The relation $x>y$ holds if every component of $x$ is equal to or greater than the corresponding component of $y$, and if at least one of those of $x$ is strictly greater than that of $y ; x \gg y$ if every component of $x$ is strictly greater than the corresponding component of $y ; x \succ_{t} y$ if $x \succsim_{t} y$, but not $y \succsim_{t} x$.

The traditional convexity assumption - also known as diminishing (i.e., nonincreasing) marginal rate of substitution-states that if $x z_{t} y$, then any weighted average (convex combination) of $x$ and $y$ is also preferred or indifferent to $y$; that is, $r x+(1-r) y \succsim_{t} y$ where $0 \leqslant r \leqslant 1$.

Each trader has an initial endowment bundle $i_{t}$. For all $t$, assume $i_{t}>0 .{ }^{5}$ That is, every trader starts with a positive amount of at least one commodity.

Assume a denumerable set of potential traders $T$, each trader labeled one of $1,2,3, \ldots$. Let $N=\left\{M: M \subset T\right.$ and $\left.\Sigma_{t \in M} i_{t} \gg 0\right\}$. A market is an element of $M$ of $N$, and by the number of traders in the market, $m$, is meant the number of elements in $M$. The process of allowing $m$ to become infinite is understood to mean considera-

${ }^{3}$ By this I mean a class of relationships between comodities which, to the best of my knowledge, has not been dealt with by economists, presumably because it is inconsistent with the assumption of convexity. The situation obtains between good $A$ and good $B$ when the simultaneous use of $a$ of $A$ and $b$ of $B$ would yield less satisfaction to the consumer than would the use of one or either of $a$ of $A$, or $b$ of $B$. Examples might well be pep pills vs. sleeping pills, ear plugs vs. a phonograph record, appetite depressant drugs vs. chocolate cake, Danish modern furnishings vs. Louis XVI. The practical problem is not difficult if one can store one of the commodities for future use with undiminished quality or if we have free disposability, but if the goods in question are time dated, as is standard practice in general equilibrium theory, then non-convexities in the preference relation will definitely result.

${ }^{4}$ A good, rigorous discussion of the nature and significance of convexity assumptions can be found in $[8]$.

${ }^{5}$ The symbol 0 is used to indicate both the real number 0 and the $n$-dimensional vector, all of whose coordinates are 0 . Context should make clear which is intended. 
tion of an arbitrarily large market or, alternatively, a sequence of markets, the number of elements in the successive markets increasing without bound. In order to make the treatment as general as possible, there are no restrictions on the order in which the traders are taken, nor is it required that the above mentioned successive markets be contained in their successors. The limits achieved in the sequel could be somewhat stronger, were one to be less general on this point.

\section{SYNTHETIC CONVEX EQUILIBRIUM}

A set is convex if the straight line segment joining any two points of the set lies entirely within the set. The convex hull of a set of points is the closure of the intersection of all the convex sets containing the set in question. Since the intersection of convex sets is also convex, the convex hull may be thought of as the "smallest" closed convex set containing the original set. From the linear character of the definition of convexity it follows that, intuitively, the convex hull of a set is nothing more than the original set with a hyperplanar segment added to the boundary wherever the set fails to be convex. In effect, we have boarded up the holes. Denote by $A_{t}(x)$ the set $\left\{y: y \succsim_{t} x\right\}$ and by $A_{t}^{\prime}(x)$ the convex hull of this set.

An assumption peculiar to treatments of non-convexity is required to make the rest of the discussion meaningful. This is the assumption of spannability, originating with Shapley and Shubik [12].

Ass Umption B - SPAnNability: For any $t \in T, w \in \Omega$, let $x \in A_{t}^{\prime}(w)$. Then there is a set of no more than $n+1$ points $x_{i}$ of $A_{t}(w)$ such that $x=\Sigma \lambda_{i} x_{i}$ where $\lambda_{i} \geqslant 0$ all $i$, and $\sum \lambda_{i}=1{ }^{6}$

Economically meaningful conditions which imply Assumption B are investigated in Appendix 3.

Any point on the boundary of $A_{t}^{\prime}(x)$ is the convex combination of points of the boundary of $A_{t}(x)$. To prove this latter statement, consider $y$ in the boundary of $A_{t}^{\prime}(x)$. Suppose $u$ does not belong to the boundary of $A_{t}(x)$. Then any point within some $\varepsilon>0$ of $u$ is a point of $A_{t}(x)$. Then if $y=r u+s v+\ldots$, any point within $r \varepsilon$ of $y$ is a point of $A_{t}^{\prime}(x)$. The contradiction shows that $u, v, \ldots$, must be points of the boundary of $A_{t}(x)$.

To each $t$ in $T$ we now assign a synthetic convex preference relation $\succsim_{k t}$.

DEFINITION: $x \succsim_{k t} y$ if and only if for all $w$ in $\Omega, x \in A_{t}^{\prime}(w)$ whenever $y \in A_{t}^{\prime}(w)$.

The relation $\succsim_{k t}$ is a transitive, reflexive, complete, closed, convex preference relation.

Consider the pure exchange economy defined by $m$ traders in $M \in N$, each with

${ }^{6} \mathrm{I}$ am indebted to Dr. Shapley for pointing out to me the importance of this assumption. 
an initial endowment $i_{t}$, and a preference relation $\succsim_{k t}$. We require the following assumption.

ASSUMPTION C-IRREDUCIBILITY OF THE CONVEX MARKET: If $I_{1}$ and $I_{2}$ are disjoint nonempty subsets of $M \in N, I_{1} \cup I_{2}=M$, and $\Sigma_{t \in I_{1}} x_{t} \leqslant \Sigma_{t \in M} i_{t}$, then there is $y \leqslant \Sigma_{t \in I_{2}} i_{t}$ such that $y=\Sigma_{t \in I_{1}} x_{t}^{*}-\Sigma_{t \in I_{1}} x_{t}$ and $x_{t}^{*} \succsim_{k t} x_{t}$ for all $t \in I_{1}$ and $x_{t}^{*} \succ_{k t} x_{t}$ for at least one $t \in I_{1}$.

Assumption $\mathrm{C}$ can be derived from the irreducibility of the non-convex market, though the converse is not generally true. The reader may satisfy himself of this merely by noting that $x_{t}^{*}$ is in $A_{t}^{\prime}(z)$ whenever it is in $A_{t}(z)$, and that $x_{t}^{*}$ is interior to $A_{t}^{\prime}(z)$ whenever it is interior to $A_{t}(z)$.

We assume that it has been shown (see Appendix 1) that there exists an $n$-dimensional vector $p$ which constitutes a price system such that excess demand is nil in all markets. There are certain other relevant facts about the traditional existence of general equilibrium to be adduced here. For each $t$ in the market, there is an equilibrium consumption vector $x_{t}$ with the property that it belongs both to a preference set and a budget set. That is, $x_{t} \gtrsim_{k t} x$ for all $x \in B_{t}(p)$ where $B_{t}(p)=\{x$ : $\left.p \cdot x=p \cdot i_{t}\right\}$. One must insist on the feasibility constraint, $\Sigma_{t \in M} x_{t}=\Sigma_{t \in M} i_{t}$.

\section{THE ROTHENBERG EQUILIBRIUM}

The question now is how close to the convex case can we stay if we reintroduce the not necessarily convex preferences with which we started? We noted in Section 3 that any point on the boundary $A_{t}^{\prime}(x)$ is the convex combination of points of the boundary of $A_{t}(x)$. Of course, this includes the possibility that the point in question is a point of the boundary of $A_{t}(x)$ to start with. Consider $x_{t}$. We know that it is on the boundary of $A_{k t}\left(x_{t}\right)$, and is therefore the convex sum of points of the boundary of $A_{t}(x)$ for some $x$ such that $A_{t}^{\prime}(x)=A_{k t}\left(x_{t}\right)$. Choose any such set of points $x_{t}^{(1)}, x_{t}^{(2)}, \ldots, x_{t}^{(n+1)}$. By Assumption B there is a set of no more than $n+1$ of these; there may be as few as one point. It is clear that since $B_{t}(p)$ is tangent to $A_{k t}\left(x_{t}\right)$ at $x_{t}$, it is also tangent to $A_{k t}\left(x_{t}\right)$ at $x_{t}^{(1)}, x_{t}^{(2)}, \ldots$ Hence, $B_{t}(p)$ is also tangent to $A_{t}(x)$ at these points. Define $z_{t}^{(1)}=x_{t}^{(1)}-x_{t}$, and in general $z_{t}^{(i)}=x_{t}^{(i)}-x_{t}$. Note that

$$
\begin{aligned}
& x_{t}=\Sigma a_{t}^{i} x_{t}^{(i)}, \text { where } a_{t}^{i}>0, \Sigma a_{t}^{i}=1 ; \\
& z_{t}^{(i)}=x_{t}^{(i)}-x_{t} ;
\end{aligned}
$$

thus,

$$
\Sigma a_{t}^{i} z_{t}^{(i)}=0 .
$$

Given $p$, for each $t$ in $M$, define $E_{t}(p)$ to be $\left\{z_{t}^{(i)}\right\}$. If $S$ is a set of points in $E^{n}$, let $C H(S)$ denote the convex hull of $S$. Then it has just been shown that $0 \in C H\left(E_{t}(p)\right)$. At this point it is necessary to call into play the friction that has traditionally 
been built into perfectly competitive systems. There would be no problem if, as in $[1,2]$, the traders were infinitesimally small. In the present model the friction, the lack of a completely smoothly working market, is interpreted as a device for overcoming the lumpiness of the traders.

The simplest rigorous way to handle the problem is to define a Rothenberg equilibrium as an assignment $\bar{x}_{t}$ of commodity bundles to traders under a price system, $p$, such that for each $t \in M \in N, p \cdot i_{t}=p \cdot \bar{x}_{t}$, and such that for no more than $n-1$ traders does it fail to be true that $\bar{x}_{t} \succsim_{t} x$ for all $x \in B_{t}(p) .{ }^{7}$ This approach has certain obvious intuitive disadvantages. It saddles a few traders with the unpleasantness associated with the non-convexities of the whole society. Moreover, in an operational sense there is no obvious means by which they can be made to act appropriately in the market. It seems ultimately to depend on just what sort of system of precedence is set up among the traders.

Lemma 1: If $S_{1}, \ldots, S_{m}$ are compact sets in $E^{n}$, and if $0 \in C H\left(S_{i}\right), i=1, \ldots, m$, then $0 \in C H\left(S_{1}+\ldots+S_{m}\right)$.

Proof: See [5, page 35].

Theorem 1: Under Assumptions $A, B$, and $C$, there exists a Rothenberg equilibrium in any $M \in N$.

Proof: By Lemma 1, $0 \in C H\left(\Sigma E_{t}(p)\right)$. From the Corollary to the ShapleyFolkman Lemma 2 (see Appendix 2), there exists $y_{t} \in C H\left(E_{t}(p)\right)$ such that $\Sigma y_{t}=0$ and at most $n-1 y_{t}$ 's (since the budget hyperplane is an $n$-1-dimensional subspace of $E^{n}$ ) are not in $E_{t}(p)$. Let $\bar{x}_{t}=x_{t}+y_{t}$. Then with at most $n-1$ exceptions $\bar{x}_{t}=x_{t}^{(i)}$ for some $i$, and $\bar{x}_{t}$ is maximal under budget constraint with respect to $\succsim_{t}$. Further, $\Sigma \bar{x}_{t}=\Sigma\left(x_{t}+y_{t}\right)=\Sigma x_{t}+\Sigma y_{t}=\Sigma x_{t}+0=\Sigma i_{t}$, so the assignment is feasible. Q.E.D.

In general, it is not true that Rothenberg equilibria are Pareto optimal.

\section{THE $\varepsilon$-EQUILIBRIUM}

Let $z=\Sigma_{t \in M} z_{t}$, where one $z_{t} \in E_{t}(p)$ is chosen for each $t \in M \in N$. The term $z$ is an expression of the social divergence from a convex preference equilibrium. In order to get an upper bound on $|z|$ when the $z_{t}$ are appropriately chosen, we apply the corollary to the Shapley-Folkman Theorem (see Appendix 2).

Definition: If $S$ is a compact subset of $E^{n}$, the radius of $S$ is

$$
\operatorname{rad}(S)=\inf _{y \in E^{n}} \sup _{a \in S}|y-a| .
$$

${ }^{7}$ This is a generalized form of the solution which J. R. Rothenberg develops in [10], in particular, on page 447 . 
The expression $\operatorname{rad}(S)$ is the radius of the smallest closed ball containing $S$.

Let $x \in C H(S)$ and let $\mathscr{L}(x, S)=\{A \mid A \subseteq S$ and $x \in C H(A)\}$.

DEFINITION : The inner radius, $r(S)$, of $S$, a compact subset of $E^{n}$, is

$$
\rho(S)=\sup _{x \in C H(S)} \inf _{A \in \mathscr{L}(x, S)} \operatorname{rad}(A)
$$

if this quantity is greater than zero; $r(S)=\sigma>0, \sigma$ small, if $\rho(S)=0$. The term $\rho(S)$ is the radius of the smallest sphere which can contain a set of points of $S$ spanning an arbitrary point of $\mathrm{CH}(\mathrm{S})$.

$$
\text { Assumption D: } L=\sup _{t \in T} \sup _{p \in \Omega} r\left(E_{t}(p)\right) \text { exists and is finite. }
$$

What Assumption D says in effect is that infinite non-convexities in the preference relations as the budget hyperplane becomes arbitrarily steep are not admissible, though an arbitrarily large number of bounded non-convexities (such as might be due to indivisibility) are admissible.

TheOREM 2:Under Assumptions $A, B, C$, and $D$, there is a choice of $z_{t}, t \in M \in N$, such that $|z| \leqslant L \sqrt{ }(n-1)$.

Proof: It was shown in Section 4 that $0 \in C H\left(E_{t}(p)\right)$. Thus, by Lemma 1, $0 \in C H\left(\Sigma_{t} E_{t}(p)\right)$. Apply the corollary to the Shapley-Folkman Theorem (Appendix $2)$; with $x=0, S_{t}=E_{t}(p)$, whence for any $p$ there is a choice of $z_{t} \in E_{t}(p)$ such that $\left|\Sigma z_{t}\right|^{2} \leqslant R^{*}$, where $R^{*}$ is the sum of the min $(m, n-1)$ largest $r^{2}\left(E_{t}(p)\right)$, which, by Assumption $\mathrm{D}$ is less than or equal to $(n-1) L^{2}$. The reduction to $n-1$ is possible because the budget hyperplane is an $n$-1-dimensional subspace of $E^{n}$. Q.E.D.

We seek now on the basis of Theorem 2 to establish a quasi-equilibrium - that is, a configuration a negligible distance from equilibrium - essentially by allowing traders to maximize and then subtracting fractions of $z$ from their consumptions. The only reason this requires some care is that $z$ may have a positive component in some commodity of which some trader has a zero consumption. In such a case it is not sufficient to assign him $\bar{x}_{t}=x_{t}+z_{t}-z / m$. The situation may arise that there is only one trader with a positive maximizing consumption of some commodity, in which case he will be forced to have the full component of $z$ in that commodity subtracted from his maximizing consumption if that component is positive. There is no assurance, even as the number of traders is made large, that there will be more than one trader with a positive maximizing consumption of a given commodity. Note that if the fashion in which the number of traders becomes large is made more specific, this last difficulty can be eliminated; e.g., replication of the 
market. Assign to each $t \in M, \bar{x}_{t}=x_{t}+z_{t}-e_{t}$, where $\Sigma e_{t}=z, p \cdot e_{t}=0$, and the sign of $e_{t}^{i}$ is the same as that of $z^{i}$, where the superscript indicates the component of the vector in $E^{n}$, and where the $e_{t}$ are chosen such that $\bar{x}_{t}$ has no negative components. This can be done since $\Sigma_{t \in M}\left(x_{t}+z_{t}\right) \geqslant \Sigma z_{t}$. Because of the fashion in which the $e_{t}$ are specified, $|z|^{2} \geqslant \Sigma\left|e_{t}\right|^{2}$.

Definition: An $\varepsilon$-equilibrium is a feasible assignment of bundles, $\bar{x}_{t}, p \cdot \bar{x}_{t}=p \cdot i_{t}$, to traders under a price system $p$ such that for each $t$ in the market there is a vector $x_{t}^{\prime}, x_{t}^{\prime}$ maximal with respect to $\gtrsim_{t}$ under the budget constraint $p \cdot x_{t}^{\prime}=p \cdot i_{t}$, for which

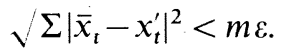

We have thus established the following theorem.

Theorem 3: Under Assumptions $A, B, C$, and $D$ on preferences for any $\varepsilon>0$, there exists an $\varepsilon$-equilibrium for sufficiently large $m$, in particular for all $m>L \sqrt{(n-1)} / \varepsilon$.

There are, of course, optimal and non-optimal $\varepsilon$-equilibria. An $\varepsilon$-equilibrium is definitely within $L \sqrt{ }(n-1)$ of an optimal assignment (though perhaps not a feasible one), but this is not saying a great deal since $L \sqrt{ }(n-1)$ may be quite large. However, some sort of $\varepsilon$-optimality may be significant for $\varepsilon$ small. Indeed, a concept of $\varepsilon$ optimality is embodied in [12].

If, instead of putting the burden of the friction in the system (that is, the $\varepsilon$ ) on the trader, one made feasibility flexible, this too would establish a quasi-equilibrium. For instance, if one defined as quasi-feasible an assignment $\bar{x}_{t}$ such that $\left|\Sigma i_{t}-\Sigma \bar{x}_{t}\right|<\varepsilon m$ for some arbitrarily chosen $\varepsilon$, then for $m$ large enough there exists a quasi-feasible assignment under which all traders can maximize satisfaction under budget constraint.

\section{THE $\varepsilon$-VALUE-EQUILIBRIUM}

If $u$ and $v$ are vectors in $E^{n}$, define $u \times v$ as the vector $\left(u^{1} v^{1}, \ldots, u^{i} v^{i}, \ldots, u^{n} v^{n}\right)$, where $u^{i}, v^{i}$ are the $i$ th components of $u, v$ respectively. The reader may satisfy himself that the operation $x$ is commutative, associative, and distributive over addition. Let $F_{t}(p)=\left\{w: w=p \times z, z \in E_{t}(p)\right\}$. An element of $F_{t}(p)$ is an expression in pecuniary terms of the corresponding element of $E_{t}(p)$. Conceptually, a raw element of $E_{t}(p)$ may be inadequate since it gives no indication of the values involved. A formulation weighting commodities by their prices gives an interpretation of the divergence from maximization, $z$, corresponding to the interpretation of transferable utility as money in [12].

Since $0 \in C H\left(E_{t}(p)\right)$, the distributive property of $\times$ implies that $0 \in C H\left(F_{t}(p)\right)$. Applying the Corollary to the Shapley-Folkman Theorem to $F_{t}(p)$, under less restrictive assumptions than were necessary to establish the $\varepsilon$-equilibrium, one can prove the existence of an $\varepsilon$-value-equilibrium. 
Definition: An e-value-equilibrium is a feasible assignment of bundles, $\bar{x}_{t}$, $p \cdot \bar{x}_{t}=p \cdot i_{t}$, to traders under a price system $p$ such that for each $t$ in the market there is a vector, $x_{t}^{\prime}, x_{t}^{\prime}$ maximal with respect to $\succsim_{t}$ under the budget constraint $p \cdot x_{t}^{\prime}=$ $p \cdot i_{r}$, for which it holds that

$$
\sqrt{\Sigma \frac{\left|p \times \bar{x}_{t}-p \times x_{t}^{\prime}\right|^{2}}{|p|^{2}}}<m \varepsilon .
$$

In order to prove the existence of the $\varepsilon$-value-equilibrium, Assumption $D$ is not necessary (though it is sufficient). Instead, postulate

Assumption D':

$$
\sup _{t \in T} \sup _{p \in \Omega} \frac{r\left(F_{t}(p)\right)}{|p|}=K
$$

exists and is finite.

What Assumption $\mathrm{D}^{\prime}$ says, in effect, is that the value of any individual's nonconvexity is bounded and that there is an upper bound on this quantity for the set of all traders. One can derive this from an assumption that the value of any individual's endowment is bounded from above. Let $w=\Sigma w_{t}$, where $w_{t} \in F_{t}(p), t \in M \in N$.

TheOREM 4:Under Assumptions $A, B, C$, and $D^{\prime}$, there is a choice of $w_{t}, t \in M \in N$, such that $|w| /|p| \leqslant K \sqrt{ }(n-1)$. Further, for any $\varepsilon>0$ there exists an $\varepsilon$-value-equilibrium for sufficiently large $m$, in particular for all $m>K \sqrt{(n-1)} / \varepsilon$.

Proof: $0 \in C H\left(F_{t}(p)\right)$ implies that $0 \in C H\left(\Sigma F_{t}(p)\right)$. Applying the corollary to the Shapley-Folkman Theorem, we have that there is $w \in \Sigma F_{t}(p)$ such that $|w| /|p| \leqslant$ $K \sqrt{ }(n-1)$. The radicand is $(n-1)$ since the budget hyperplane is an $(n-1)$-dimensional subspace of $E^{n}$. To each $t \in M$, assign $\bar{x}_{t}=x_{t}+y_{t}-e_{t}$ where $w_{t}=p \times y_{t}, p \cdot e_{t}=0$, $\Sigma e_{t}=\Sigma y_{t}$, and sign $e_{t}^{i}=\operatorname{sign} \Sigma e_{t}^{i} . \bar{x}_{t}$ can be kept in $\Omega$ since $\Sigma\left(x_{t}+y_{t}\right) \geqslant \Sigma y_{t}$. It suffices that

$$
\sqrt{\sum_{t \in M} \frac{\left|p \times e_{t}\right|^{2}}{|p|^{2}}} \leqslant \frac{|w|}{|p|}
$$

Lemma 2: If $u$ and $v$ are vectors in $E^{n},|u \times v| \leqslant|u||v|$.

PROOF: $|u|^{2}|v|^{2}=\left(\Sigma u^{i 2}\right)\left(\Sigma v^{i 2}\right) \geqslant \Sigma u^{i 2} v^{i 2}=|u \times v|^{2}$.

Corollary 1: Assumption D implies assumption $D^{\prime}$.

Proof: For any $t, p$, consider $z_{t} \in E_{t}(p)$. From the lemma we have $p \times z_{t} \leqslant|p|\left|z_{t}\right|$. 
Therefore, $r\left(F_{t}(p)\right) \leqslant|p| r\left(E_{t}(p)\right)$. By Assumption D the appropriate suprema exist on the right and hence they exist on the left as well.

Q.E.D.

COROllary 2: Any E-equilibrium is an E-value-equilibrium.

Proof: $\sqrt{ }\left(\Sigma\left|\bar{x}_{t}-x_{t}^{\prime}\right|^{2}\right) \leqslant m \varepsilon$. By the distributive property of $\times$ and the lemma, we have

$$
\sqrt{\sum \frac{\left|p \times \bar{x}_{t}-p \times x_{t}^{\prime}\right|^{2}}{|p|^{2}}}<m \varepsilon
$$

\section{RELATIONS WITH THE LITERATURE}

The reader is probably already quite aware of the classic proofs of the existence of competitive equilibrium with convex preferences. There have been several heuristic treatments by economists of markets with non-convex preferences in two dimensions (see $[3,4,7,10])$. In any rigorous sense, however, the question of nonconvexity has been dealt with much better by game theorists than by economists.

There have been two fairly general approaches using game theoretical techniques not so abstruse as to obscure the conclusions for the economist, originating with Aumann $[1,2]$ and Shapley and Shubik [12]. Both use core analysis. The core of a game - in this case a market game - is the set of all those outcomes which no player or coalition of players can profitably prevent. In general, the competitive equilibrium, when it exists, is contained in the core; there may be points in the core unattainable as competitive equilibria.

Professor Aumann assumes a market with uncountably many traders. Under these conditions, and various other fairly traditional assumptions on the preference sets (not including convexity), he shows that the core coincides with the set of competitive equilibria. Moreover, he goes on to show the existence of such equilibria. Professor Aumann's model is certainly a mathematically elegant approach to the question of perfect competition. Aumann's model, however, has an aspect that is conceptually distressing, though mathematically necessary. For though the number of traders is uncountable, the quantity of commodities is finite, thus leaving almost all traders with literally next to nothing. Though it seems reasonable to treat an individual as $5 \times 10^{-9}$ of the United States economy, I find it difficult to conceive of him as 0 of it. Nevertheless, the use of uncountably many traders is certainly a simple and admirable device for embodying the concept of perfect competition and for providing the friction in the system necessary to assure existence even with non-convexities. With respect to existence, it seems to me that the results of this paper as $m \rightarrow \infty$ are conceptually, though not mathematically, equivalent to Aumann's [2].

A less elegant but also less abstruse approach is provided by Shapley and Shubik 
in their device of the $\varepsilon$-core. Essentially, the $\varepsilon$-core is the set of all those solutions that cannot be blocked by any coalition at a profit of more than $\varepsilon$ (in cardinal utility terms) to the coalition (strong $\varepsilon$-core), or to each member of the coalition (weak $\varepsilon$-core). For sufficiently large $m$, under certain not terribly restrictive assumptions, both strong and weak $\varepsilon$-cores can be shown to exist. One suspects that for appropriately defined $f$, if $f(|z|)<m \varepsilon$, the $\varepsilon$-equilibrium of divergence less than $|z|$ is contained in the strong $m \varepsilon$-core. For appropriately defined $g$, one suspects that if $g(|z| / m)<\varepsilon$, then the $\varepsilon$-equilibrium with individual divergence less than $|z| / m$ is contained in the weak $\varepsilon$-core. It is awkward from the economist's point of view that some of the assumptions of the Shapley-Shubik paper are comparatively strong. Of these, the assumption that everyone has the same utility function is merely a matter of convenience. The assumption of transferable utility (this does not include an interpersonal comparison, but rather supposes that there exists some goodcalled "money" by Shapley and Shubik - for which each trader has a constant marginal utility) is surely less strong, but also less expendable. One indication of the parallel nature of the $\varepsilon$-core and the $\varepsilon$-equilibrium is that the Shapley-Shubik use of the concave majorant game is parallel in context to the use here of the synthetic convex equilibrium.

\section{Stanford University \\ and}

The RAND Corporation

\section{APPENDIX 1}

Proof of Applicability of the McKenzie Proof to the Convex Case

The McKenzie proof of the existence of competitive equilibrium [9] requires six assumptions on the possible net consumptions of traders, possible productions, and the relations between these. We seek to show that these are satisfied by the pure exchange economy described above.

For each trader $t$, McKenzie assumes $X_{t}$ a set of possible net trades fulfilling the following:

Assumption 1: $X_{t}$ is closed, convex, and bounded from below.

Assumption 2: $X_{t}$ is completely ordered by a convex and closed preference relation.

Let $X_{t}=\left\{x: \Sigma_{r \in M \sim\{t\}} i_{r} \geqslant x \geqslant-i_{t}\right\}$. That is, $X_{t}$ is the set of possible net trades which leave $t$ with an amount more than or equal to none of each commodity. Thus $X_{t}$ fulfills Assumption 1, and when we define the preference relation $x(\geqslant) y$ to hold if and only if $x+i_{t} \succ_{k t} y+i_{t}$ holds, then we have an appropriate closed convex preference relation.

Since no production goes on in this model, the possible set of net production vectors is $Y=\{0\}$. This clearly satisfies

Assumption 3: $Y$ is a closed convex cone.

AsSUMPTION 4: $Y \cap \Omega=\{0\}$.

A point $x$ is relative interior to a set $X$ if $x$ is interior to $X$ in the smallest flat containing $X$. A flat is a 
set of vectors $F$ such that $x$ and $x^{\prime}$ in $F$ implies $t x+(1-t) x^{\prime}$ in $F$ for all real $t$. Thus, the smallest flat containing $Y$ is $\{0\}=Y$ and 0 is interior to $Y$ in $\{0\}=Y$. Thus, we satisfy

Assumption 5: $X_{\mathrm{t}} \cap Y \neq 0$. Moreover, there is a common point in the relative interiors of $Y$ and $X$. $\left(X=\Sigma X_{t}\right)$.

The sixth assumption is the irreducibility of the market: "However we may partition the consumers into two groups if the first group receives an aggregate trade which is an attainable output for the rest of the market, the second group has within its feasible aggregate trades one which, if added to the goods already obtained by the first group, can be used to improve the position of someone in that group while damaging the position of none there" (p. 59). This is Assumption C.

Under assumptions 1-6, McKenzie proves the existence of a price vector $p \in \Omega$ and an assignment $x_{t}$ of goods to traders satisfying appropriate maximization and feasibility conditions. As shown, this proof and its conclusions are applicable to the pure exchange, convex preference model of Section 3 .

\section{APPENDIX 2}

\section{The Shapley-Folkman Theorem}

As it is not generally available, the statement and proof of the Shapley-Folkman theorem are included here. With the exception of the final corollary (due to myself), this treatment is taken with slight alterations in notation from Shapley and Folkman's [11].

If $S_{1}$ and $S_{2}$ are any sets in $E^{n}$, then $S_{1}+S_{2}$ will denote the set of points $z=x+y$, where $x \in S_{1}, y \in S_{2}$.

If $C$ is compact and convex, and if $x$ is in $C$, then the "convex face" of $x$ is defined as the largest convex subset $K$ of $C$ such that $x$ is in the relative interior of $K$. The "facial dimension" of $x$, denoted con $(x)$, is the dimension of the convex face of $x$. In particular, con $(x)=0$ if and only if $x$ is an extreme point. $^{8}$

Lemma 1: Let $S$ be a compact set in $E^{n}$. Let $x \in C H(S)$. Then there is an $a \in S$ such that $\|x-a\| \leqslant \operatorname{rad}(S)$. In other words,

$$
\max _{x \in C H(S)} \min _{a \in S}\|x-a\| \leqslant \min _{x \in E^{n}} \max _{a \in S}\|x-a\| .
$$

Proof: Let $c$ minimize $\max _{a \in S}\|c-a\|$. If $x=c$, then any $a \in S$ will satisfy the lemma. If $x \neq c$, then we have $(x-c) \cdot(x-a) \leqslant 0$ for at least one $a \in S$, since a weighted average of such terms is zero. But then

$$
\operatorname{rad}^{2}(S) \geqslant\|a-c\|^{2}=\|a-x\|^{2}+\|x-c\|^{2}-2(x-c) \cdot(x-a) \geqslant\|a-x\|^{2} . \quad \text { Q.E.D. }
$$

Lemma 2: Let $S_{i}, i=1, \ldots, m$, be compact sets in $E^{n}$, let $m>n$, and let

$$
x \in C H\left(S_{1}+\ldots+S_{m}\right) .
$$

Then for some $i^{*}$ and $a$, we have $a \in S_{i^{*}}$ and

$$
x-a \in C H\left(S_{1}+\ldots+S_{i^{*-1}}+S_{i^{*}+1}+\ldots+S_{m}\right) .
$$

COROLlaRY: Under the same hypothesis, there exist $y_{i} \in C H\left(S_{i}\right), i=1, \ldots, m$, such that $\Sigma y_{i}=x$ and such that with at most $n$ exceptions, $y_{i} \in S_{i}$.

Proof: The Corollary follows from the lemma by a simple induction. To prove the lemma, find $x_{i} \in C H\left(S_{i}\right), i=1, \ldots, m$, such that $\Sigma x_{i}=x$, and such that $\Sigma$ con $\left(x_{i}\right)$ is minimized. Assume that $x_{i} \notin S_{i}$, all $i$.

${ }^{8}$ See $[6$, pp. 6, 7] for a fuller description of $K$ and $\operatorname{con}(x)$. 
Then, for each $i$, con $\left(x_{i}\right)>0$. Let $a_{i}$ be any element of $S_{i}$ that lies in the convex face of $x_{i}$. If $L_{i}$ is the line through $a_{i}$ and $x_{i}$, then we see that $x_{i}$ is in the interior of the interval $L_{i} \cap C H\left(S_{i}\right)$.

Since $n<m$, the family of vectors $\left\{a_{i}-x_{i}\right\}$ is linearly dependent, and constants $\left\{c_{i}\right\}$ exist, not all zero, such that $\Sigma c_{i}\left(a_{i}-x_{i}\right)=0$.

Define

$$
x_{i}(t)=x_{i}+c_{i}\left(a_{i}-x_{i}\right) t
$$

Then $\Sigma x_{i}(t)=x$; moreover, in a neighborhood of $t=0$ we have $x_{i}(t) \in C H\left(S_{i}\right)$, all $i$. Let $t_{0}$ be the largest value of $t$ such that $x_{i}(t) \in C H\left(S_{i}\right)$, all $i$, and define $x_{i}^{\prime}=x_{i}\left(t_{0}\right), i=1, \ldots, m$. Then, for at least one $i, x_{i}^{\prime}$ is not in the relative interior of the convex face of $x_{i}$, and we have $\Sigma$ con $\left(x_{i}^{\prime}\right)<\Sigma$ con $\left(x_{i}\right)$, a contradiction. It follows that $x_{i} \in S_{i}$ for at least one $i$, and this $i, x_{i}$ can be taken for the $i^{*}, a$ of the lemma.

Q.E.D.

Lemma 3: Let $x \in C H\left(S_{1}+\ldots+S_{m}\right)$. Then there are $a_{i} \in S_{i}, i=1, \ldots, m$, such that

$$
\left\|x-\Sigma a_{i}\right\|^{2} \leqslant \Sigma \operatorname{rad}^{2}\left(S_{i}\right) .
$$

Proof: We proceed by induction on $m$. If $m=1$, Lemma 1 applies. Assume that the lemma has been proved for some fixed $m \geqslant 1$, and let $y \in C H\left(S_{1}+\ldots+S_{m+1}\right)$. Choose $x, z$ such that $x \in C H\left(S_{1}+\ldots+S_{m}\right)$, $z \in C H\left(S_{m+1}\right), y=x+z$. We can then find $a_{i} \in S_{i}, i=1, \ldots, m$, such that

$$
\|x-a\|^{2} \leqslant \sum_{1}^{m} \operatorname{rad}^{2}\left(S_{i}\right)
$$

where $a=\Sigma_{1}^{m} a_{i}$.

Choose $w_{0}$ in $C H\left(S_{m+1}\right)$ so as to minimize $\left\|y-a-w_{0}\right\|$. Then we have

$$
\left\|y-a-w_{0}\right\|^{2} \leqslant\|y-a-z\|^{2}=\|x-a\|^{2} \leqslant \sum_{1}^{m} \operatorname{rad}^{2}\left(S_{i}\right) .
$$

Moreover, for any $w \in C H\left(S_{m+1}\right)$ and for any $0<t<1$,

$$
\left\|y-a-w_{0}\right\|^{2} \leqslant\left\|y-a-t w-(1-t) w_{0}\right\|^{2} .
$$

If we let $t \rightarrow 0^{+}$, this yields the linear inequality

$$
\left(y-a-w_{0}\right) \cdot\left(w-w_{0}\right) \leqslant 0,
$$

valid for all $w$ in $C H\left(S_{m+1}\right)$. Since equality holds at $w=w_{0}$, it also holds on the convex face of $w_{0}$. and hence on a subset $T$ of $S_{m+1}$ such that $w_{0} \in C H(T)$. Using Lemma 1, take $a_{m+1} \in T$ with $\left\|a_{m+1}-w_{0}\right\|$ $\leqslant \operatorname{rad}(T) \leqslant \operatorname{rad}\left(S_{m+1}\right)$. Then $\left(y-a-w_{0}\right) \cdot\left(a_{m+1}-w_{0}\right)=0$, and we have

$$
\begin{aligned}
\left\|y-\left(a+a_{m+1}\right)\right\|^{2} & =\left\|\left(y-a-w_{0}\right)-\left(a_{m+1}-w_{0}\right)\right\|^{2} \\
& =\left\|y-a-w_{0}\right\|^{2}+\left\|a_{m+1}-w_{0}\right\|^{2}-2\left(y-a-w_{0}\right) \cdot\left(a_{m+1}-w_{0}\right) \\
& \leqslant \sum_{i=1}^{m+1} \operatorname{rad}^{2}\left(S_{i}\right) .
\end{aligned}
$$

THEOREM: Let $S_{1}, \ldots, S_{m}$ he compact sets in $E^{n}$. and let $x \in C H\left(S_{1}+\ldots+S_{m}\right)$. Then there are points $a_{i} \in S_{i}, i=1, \ldots, m$, such that $\left\|x-\sum a_{i}\right\|^{2} \leqslant R$, where $R$ is the sum of the min $(m, n)$ largest $\operatorname{rad}^{2}\left(S_{i}\right)$.

ProOF: If $n \geqslant m$, then Lemma 3 applies. If $n<m$, apply the corollary to Lemma 2 to obtain $\left\{y_{i}\right\}$ and

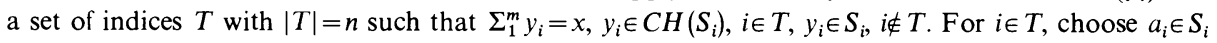
so that

$$
\left\|\sum_{T} a_{i}-\sum_{T} y_{i}\right\|^{2} \leqslant \operatorname{rad}^{2}\left(S_{i}\right) \leqslant R
$$

(using Lemma 3). For $i \notin T$, choose $a_{i}=y_{i} \in S_{i}$.

Q.E.D. 
Corollary to the Shapley-Folkman Theorem: ${ }^{9}$ Let $S_{1}, \ldots, S_{m}$ be compact sets in $E^{n}$ and let $x \in C H\left(S_{1}+S_{2}+\ldots+S_{m}\right)$. Then there are points $a_{i} \in S_{i}, i=1, \ldots, m$ such that $\left\|x-\Sigma a_{i}\right\|^{2} \leqslant R^{*}$ where $R^{*}$ is the sum of the $\min (m, n)$ largest $r^{2}\left(S_{i}\right)$.

ProOF: Since $C H\left(\Sigma S_{i}\right)=\Sigma C H\left(S_{i}\right)$ there exists $x_{i} \in C H\left(S_{i}\right), i=1, \ldots, m$, such that $\Sigma x_{i}=x$. For $i$ such that $r\left(S_{i}\right)=\operatorname{rad}\left(S_{i}\right)$ let $Q_{i}=S_{i}$. For other $i$ let $Q_{i} \subseteq S_{i}, x_{i} \in C H\left(Q_{i}\right), Q_{i}$ compact, $\operatorname{rad} Q_{i} \leqslant r\left(S_{i}\right)$. Such $Q_{i}$ exists by definition of $r\left(S_{i}\right) ; x \in C H\left(Q_{1}+Q_{2}+\ldots+Q_{m}\right)$. Apply the Shapley-Folkman Theorem to $x$, $Q_{1}, \ldots, Q_{m}$. We have that there exist $a_{i} \in Q_{i} \subseteq S_{i}$ such that $\left\|x-\Sigma a_{i}\right\|^{2}$ is less than or equal to the sum of the $\min (m, n)$ largest $\operatorname{rad}^{2}\left(Q_{i}\right)$. But $\operatorname{rad}^{2}\left(Q_{i}\right) \leqslant r^{2}\left(S_{i}\right)$ implies there exist $a_{i} \in S_{i}$ such that $\left\|x-\Sigma a_{i}\right\|^{2} \leqslant R^{*}$.

Q.E.D.

\section{APPENDIX 3}

\section{Spannability of Preference Sets ${ }^{10}$}

In some markets all the spannability required may be deducible from assumptions not directly related to those adduced here. For instance, if we know, for whatever reasons (e.g., at least one trader $t$ has a strong desirability condition and $\left.i_{t} \gg 0\right), p \gg 0$, then this condition guarantees enough spannability for the purposes of this paper. A fairly general set of individual conditions ensuring spannability even with zero prices of some goods is presented here. For convenience we drop a good deal of notation and refer to $A_{t}(w)$ as $A$.

Consider the three conditions:

$(\alpha)$ Weak desirability: If $x \geqslant y$, and $y \in A$, then $x \in A$.

( $\beta$ ) Eventual weak desirability: For some compact subset $S$ of $E^{n}$, if $y \in A, x \geqslant y, x \notin S$, then $x \in A$.

$(\gamma)$ Given any consumption there is an additional amount of any commodity which the trader would not mind having: If $y \in A$, then for $j=1, \ldots, n$, there exists a positive real number $M_{j}=M_{j}(y)$ such that if $x_{j}=y+M_{j} u_{j}$, where $u_{j}$ is the $j$ th unit vector, then $x_{j} \in A$.

Spannability can be deduced from each of these by progressively more complex arguments. Inasmuch as $(\alpha)$ implies $(\beta)$ and $(\gamma)$, and $(\beta)$ implies $(\gamma)$, we will prove only that $(\gamma)$ implies spannability. $A$ is a closed subset of the nonnegative orthant of $E^{n}$. Points in the convex hull of $A$ are either points spanned by points of $A$, or limit points of spanned points. We must show that under assumption $(\gamma)$, such a limit point is spanned by points of $A$.

Let $x \in C H(A)$. Then there is a sequence $x(k) \rightarrow x$ such that each $x(k)$ is spanned by $A$. Specifically, we have

$$
x(k)=\sum_{i=0}^{n} \lambda_{i}(k) a^{i}(k), \quad \text { where }\left\{\begin{array}{l}
\lambda_{i}(k) \geqslant 0, \quad i=0, \ldots, n, \\
\sum_{i} \lambda_{i}=1, \\
a^{i}(k) \in A, \quad i=0, \ldots, n .
\end{array}\right.
$$

(The $a^{i}(k)$ need not be distinct.)

Taking a subsequence, relabelling, and applying the Bolzano-Weierstrass theorem, we can obtain

$$
\begin{array}{ll}
\lambda^{i}(k) \rightarrow \lambda_{i} & \text { (all } i), \\
a^{i}(k) \rightarrow a^{i} & \text { (for a certain class of indices } i \text { : Class I), } \\
\left\|a^{i}(k)\right\| \rightarrow \infty & \text { (for the rest: Class II). }
\end{array}
$$

Note that $\Sigma \lambda_{i}=1$, also that $a^{i} \in A$, since $A$ is closed. Now for $i \in C$ lass II we obviously have $\lambda_{i}=0$. Hence $\Sigma_{\text {Class } 1} \lambda_{i}=1$. (In particular, Class I is not empty.) Define

$$
y=\sum_{\text {Class I }} \lambda_{i} a^{i}
$$

${ }^{9}$ I am indebted to K. J. Arrow, L. S. Shapley, W. P. Heller, and D. Starrett for constructive criticism on this corollary.

${ }^{10} \mathrm{I}$ am indebted to Dr. Shapley for his extensive work on this section. 
Now

$$
x(k)=\sum_{\mathrm{I}} \lambda_{i}(k) a^{i}(k)+\sum_{\mathrm{II}} \lambda_{i}(k) a^{i}(k) \geqslant \sum_{\mathrm{I}} \lambda_{i}(k) a^{i}(k) .
$$

Let $k \rightarrow \infty ; x \geqslant y$. Define $b^{i}=a^{i}+x-y$, all $i$ in Class I. Then $x=\Sigma_{1} \lambda_{i} b^{i}$. If we can show that the $b^{i}$ are spanned by points of $A$, then we will have proved that $x$ is spanned by points of $A$.

It follows from $(\gamma)$ that there will be arbitrarily large such numbers $M_{j}$. Given $b^{i}>a^{i}$, choose $M_{j}\left(a^{i}\right)>$ $n\left(b_{j}^{i}-a_{j}^{i}\right)$ where the subscript indicates the $j$ th component of the vector.

Define

$$
\alpha_{j}=\frac{b_{j}^{i}-a_{j}^{i}}{M_{j}} .
$$

Then $\sum_{j=1}^{n} \alpha_{j} M_{j} u_{j}=b^{i}-a^{i}$. Also

$$
0<\sum_{k=1}^{n} \alpha_{k}<\sum_{1}^{n} \frac{1}{n}=1
$$

Define

$$
d^{i}=\sum_{j=1}^{n} \frac{\alpha_{j}}{\sum \alpha_{k}}\left(a^{i}+M_{j} u_{j}\right)
$$

Then

$$
d^{i}=a^{i}+\frac{b^{i}-a^{i}}{\sum \alpha_{k}}
$$

Hence $a^{i}$ and $d^{i}$ span $b^{i}$. But $d^{i}$ is spanned by the points $\left(a^{i}+M_{j} u_{j}\right) \in A$.

Q.E.D.

\section{REFERENCES}

[1] Aumann, R. J.: "Markets with a Continuum of Traders," Econometrica, Vol. 32 (1964), 39-50.

[2] — : "Existence of Competitive Equilibria in Markets with a Continuum of Traders," Econometrica, Vol. 34 (1966), 1-17.

[3] Bator, F. M.: "Convexity, Efficiency, and Markets," Journal of Political Economy, Vol. 69 (1961), 480-83; "Rejoinder," Ibid., 489.

[4] Farrell, M. J.: "The Convexity Assumption in the Theory of Competitive Markets," Journal of Political Economy, Vol. 67 (1959), 377-91; "A Reply," and "Rejoinder," Ibid., Vol. 69 (1961), 484-89, 493.

[5] Fenchel, W.: "Convex Cones, Sets and Functions," Mimeographed, Dept. of Mathematics, Princeton University, September, 1953.

[6] Karlin, S., and L. S. Shapley: "Geometry of Moment Spaces," Memoir No. 12, American Mathematical Society, 1953.

[7] Koopmans, T. C.: "Convexity Assumptions, Allocative Efficiency, and Competitive Equilibrium," Journal of Political Economy, Vol. 69 (1961), 478-79.

[8] _ : "Allocation of Resources and the Price System," in Three Essays on the State of Economic Science, New York: McGraw-Hill, 1957.

[9] McKenziE, L. W.: "On the Existence of General Equilibrium for a Competitive Market," Econometrica, Vol. 27 (1959), 54-71.

[10] Rothenberg, J. R.: "Non-Convexity, Aggregation, and Pareto Optimality," Journal of Political Economy, Vol. 68 (1960), 435-68; “Comments on Non-Convexity,” Ibid., Vol. 69 (1961), 490-92.

[11] Shapley, L. S., and J. H. Folkman: "Starr's Problem," private correspondence, January, 1966.

[12] Shapley, L. S., AND M. Shubik: "Quasi-Cores in a Monetary Economy with Nonconvex Preferences," Econometrica, Vol. 34, No. 4 (Oct., 1966). 